\title{
INTERIOR AND BOUNDARY CONTINUITY OF WEAK SOLUTIONS OF DEGENERATE PARABOLIC EQUATIONS
}

BY

\author{
WILLIAM P. ZIEMER
}

ABSTRACT. In this paper we consider degenerate parabolic equations of the form

$$
\beta(u)_{t}-\operatorname{div} A\left(x, t, u, u_{x}\right)+B\left(x, t, u, u_{x}\right) \ni 0
$$

where $A$ and $B$ are, respectively, vector and scalar valued Baire functions defined on $U \times R^{1} \times R^{n}$, where $U$ is an open subset of $R^{n+1}(x, t)$. The functions $A$ and $B$ are subject to natural structural inequalities. Sufficiently general conditions are allowed on the relation $\beta \subset R^{1} \times R^{1}$ so that the porus medium equation and the model for the two-phase Stefan problem can be considered. The main result of the paper is that weak solutions of $(*)$ are continuous throughout $U$. In the event that $U=\Omega \times(0, T)$ where $\Omega$ is an open set of $R^{n}$, it is also shown that a weak solution is continuous at $\left(x_{0}, t_{0}\right) \in \partial \Omega \times(0, T)$ provided $x_{0}$ is a regular point for the Laplacian on $\Omega$.

0. Introduction. In this paper we consider the problem of continuity of weak solutions of parabolic equations of the form

$$
\frac{\partial}{\partial t} \beta(u)-\operatorname{div} A\left(x, t, u, u_{x}\right)+B\left(x, t, u, u_{x}\right) \ni 0
$$

where $A$ and $B$ are, respectively, vector and scalar valued Baire functions defined on $U \times R^{1} \times R^{n}$, and $U$ is an open subset of $R^{n+1}(x, t)$. Throughout this paper, $U$ will take the form $\Omega_{T}=\Omega \times(0, T]$ where $\Omega$ is an open, bounded subset of $R^{n}$. The functions $A$ and $B$ are required to satisfy the following structural inequalities.

$$
\begin{aligned}
& |A(x, t, u, p)| \leqslant a_{0}|p|+a_{1}(x, t)|u|+a_{2}(x, t), \\
& |B(x, t, u, p)| \leqslant b_{0}|p|^{2}+b_{1}(x, t)|p|+b_{2}(x, t)|u|+b_{3}(x, t), \\
& A(x, t, u, p) \cdot p \geqslant c_{0}|p|^{2}-c_{1}(x, t)|u|^{2}-c_{2}(x, t),
\end{aligned}
$$

where $a_{0}, b_{0}$ and $c_{0}$ are constants with $a_{0}$ and $b_{0}$ nonnegative and $c_{0}>0$. The remaining coefficients are nonnegative measurable functions which, for simplicity of exposition, we will assume to be bounded. Thus, we assume there is a nonnegative constant $K$ such that

$$
a_{i}(x, t) \leqslant K, \quad b_{i}(x, t) \leqslant K, \quad c_{i}(x, t) \leqslant K .
$$

Our results remain valid if the coefficients lie in those appropriate Lebesgue classes which have been considered by previous authors, e.g., [LSU, T]. Throughout, we

Received by the editors March 28, 1981

1980 Mathematics Subject Classification. Primary 35K10, 35K15; Secondary 35K20, 35K65.

${ }^{1}$ Research supported in part by a grant from the National Science Foundation. 
allow sufficiently general conditions on $\beta$ so that (1) includes both the porous medium equation and a model for the two-phase Stefan problem.

The main objective of this paper is to provide a technique that will establish continuity of weak solutions of (1) at the lateral boundary of $\Omega_{T}$ as well as at points of $\Omega_{T}$. In the case $A\left(x, t, u, u_{x}\right)=u_{x}, B=0$ and $n>1$, continuity of weak solutions was established by Caffarelli and Friedman, $[\mathbf{C F}]$, for the porous medium equation, and by Caffarelli and Evans, [CE], for the two-phase Stefan problem. P. Sacks, [S], extended the results of [CE] by considering suitable $B$ of the form $B=B(x, t)$. Recently, DiBenedetto, [D1, D2], established continuity of weak solutions of (1) in essentially the same generality considered in this paper. The approach employed by DiBenedetto is based on a parabolic version of DeGiorgi's technique as presented in [LSU]. The proof that we present here is based primarily on the Moser iteration technique as developed in [T] for quasilinear equations, and is therefore able to avoid much of the technical complexity associated with the DeGiorgi method. Our proof is also able to deal with the question of continuity of the solution at the lateral boundary of a cylindrical domain $\Omega \times(0, T]$. We are able to prove that a solution is continuous at a boundary point $(x, t)$, where $x \in \partial \Omega$ and $0<t<T$, provided $x$ is a regular point for the Laplacian on $\Omega$.

The main idea of the proof is to employ the full strength of the Moser technique, which includes the parabolic version of the John-Nirenberg Lemma, to establish that each point of the domain $\Omega \times(0, T]$ is a Lebesgue point of a weak solution. Here, the term "Lebesgue point" refers to a parabolic modification of that classical concept. Then, we appeal to a suitable version of a fundamental lemma of DeGiorgi as presented in [D1, Lemma 3.1] or [CE, Lemma 3.1] which has been adopted to reflect the singularity in the equation. This lemma implies that a weak solution is continuous at each of its Lebesgue points. This same approach is also used to establish continuity at the boundary of a cylindrical domain.

The author is pleased to acknowledge several interesting conversations with Emmanuel DiBenedetto during the preparation of this paper.

1. Preliminaries. For $\Omega$ a bounded open subset of $R^{n}$, the cylindrical domain $\Omega \times(0, T]$ will be denoted by $\Omega_{T}$ and let $\Omega(t)=\Omega \times\{t\}$. We let $x=\left(x_{1}, \ldots, x_{n}\right)$ denote points in $R^{n}$ and the gradient of a function $u$ will be denoted by $u_{x}$ or $\nabla u$. Points in $R^{n+1}$ will generally be denoted by $z=(x, t)$. Following common practice, the letter $C$ will denote a constant that may change from line to line in the same proof. We will denote by $W_{2}^{1,1}\left(\Omega_{T}\right)$, the Sobolev space of functions whose distributional first derivatives belong to $L_{2}\left(\Omega_{T}\right)$, whereas $W_{2}^{1,0}\left(\Omega_{T}\right)$ will be the subspace consisting of those functions $u$ for which $\partial u / \partial x_{i} \in L_{2}\left(\Omega_{T}\right), i=1,2, \ldots, n$. The space of those elements of $W_{2}^{1,1}\left(\Omega_{T}\right)$, whose trace on $\partial \Omega \times(0, T]$ is zero, is denoted by $W_{2,0}^{1,1}\left(\Omega_{T}\right)$. Let $V_{2}\left(\Omega_{T}\right) \subset W_{2}^{1,0}\left(\Omega_{T}\right)$ denote the Banach space with norm

$$
|u|_{V^{2}\left(\Omega_{T}\right)}=\underset{0 \leqslant t \leqslant T}{\operatorname{ess} \sup }\|u(\cdot, t)\|_{2, \Omega}^{2}+\|\nabla u\|_{2, \Omega_{T}}^{2} .
$$

Finally, let $V_{2}^{1,0}\left(\Omega_{T}\right) \subset V_{2}\left(\Omega_{T}\right)$ denote that subspace of elements $u$ for which the map $t \rightarrow u(\cdot, t)$ is continuous relative to the norm on $L_{2}(\Omega)$. 
Throughout we will be interested in $\beta$ of the following two types.

Type I. Here we assume that $\beta$ is a relation on $R^{1} \times R^{1}$ given by

$$
\beta(r)= \begin{cases}r, & r>0 \\ {[-a, 0],} & r=0 \\ r-a, & r<0\end{cases}
$$

where $a>0$. As shown in [D1] there is no loss of generality in assuming $\beta$ is of this simple form. Indeed, if $\beta$ were assumed to be given by

$$
\beta(r)= \begin{cases}\beta_{1}(r), & r>0 ; \\ {[-a, 0],} & r=0 ; \\ \beta_{2}(r)-a, & r<0 ;\end{cases}
$$

where $\beta_{i}, i=1,2$, are monotone increasing functions whose derivatives are almost everywhere bounded away from 0 and $\infty$, then the question of continuity of weak solutions of (1) easily reduces to the case of $\beta$ given by (4).

Type II. Let $\beta$ be a continuous monotone increasing function with $\beta(0)=0$ satisfying the following.

(i) Assume the upper right (Dini) derivate of $\beta$ is finite for all $r \neq 0$. Denote this derivate simply by $\beta^{\prime}(r)$.

(ii) There is a constant $\alpha_{0}$ such that

$$
\beta^{\prime}(r) \geqslant \alpha_{0}>0, \text { for all } r \neq 0 .
$$

(iii) $\liminf _{r \rightarrow 0} \beta^{\prime}(r)=\infty$.

(iv) There exists $\delta>0$ such that $\beta^{\prime}(s) \leqslant \beta^{\prime}(r)$, for $s \notin[-\delta, \delta]$ and $r \in[-\delta, \delta]-$ $\{0\}$, and $\beta^{\prime}$ is increasing on $[-\delta, 0)$ and decreasing on $(0, \delta]$.

Definition 1.1. A function $u \in W_{2}^{1,1}\left(\Omega_{T}\right)$ is called a weak solution of (1) if

(5)

$$
\begin{aligned}
-\int_{\Omega} w(x, t) & \phi(x, t) d x \int_{t_{0}}^{t_{1}}+\int_{t_{0}}^{t_{1}} \int_{\Omega}+w(x, t) \phi_{t}(x, t) \\
= & \int_{t_{0}}^{t_{1}} \int_{\Omega}+A\left(x, t, u, u_{x}\right) \cdot \nabla \phi+B\left(x, t, u, u_{x}\right) \phi(x, t),
\end{aligned}
$$

for all $\phi \in W_{2,0}^{1,1}\left(\Omega_{T}\right)$ and all intervals $\left[t_{0}, t_{1}\right] \subset(0, T]$. Here, $w$ is a function such that

$$
\left\{(z, w(z)): z \in \Omega_{T}\right\} \subset\left\{(z, \beta[u(z)]): z \in \Omega_{T}\right\},
$$

if $\beta$ is of type I. If $\beta$ is of type II, then $w=\beta(u)$.

2. Weak solutions and Lebesgue points. Since we will assume that weak solutions of (1) are bounded, it will be convenient to formulate the structure in the following way.

$$
\begin{gathered}
|A(x, t, u, p)| \leqslant a_{0}|p|+a_{1}, \quad|B(x, t, u, p)| \leqslant b_{0}|p|^{2}+b_{1}, \\
A(x, t, u, p) \cdot p \geqslant|p|^{2}-c_{1} .
\end{gathered}
$$

Choose $d$ such that $d-1=a_{1}+b_{1}+c_{1}$. 
Now consider a function $u \in W_{2}^{1,1}\left(\Omega_{T}\right)$ that is a weak solution of (1) with structure (5). In this section we will show that any point $z_{0} \in \Omega_{T}$ is a parabolic Lebesgue point for $u$. For notational simplicity, we will take $z_{0}=(0,0)$. The estimates that are obtained below are set in terms of space-time cylinders, i.e., products of $n$-balls with time intervals. Thus, we will consider cylinders of the following form:

$$
\begin{aligned}
R(r) & =B(r) \times\left(\sigma_{1} r^{2}, \sigma_{2} r^{2}\right), \\
R^{-}(r) & =B\left(r^{\prime}\right) \times\left(\tau_{1} r^{2}, \tau_{2} r^{2}\right), \\
R^{+}(r) & =B\left(r^{\prime}\right) \times\left(\tau_{3} r^{2}, \tau_{4} r^{2}\right), \\
R^{*}(r) & =B\left(r^{\prime \prime}\right) \times\left(\rho_{1} r^{2}, \rho_{2} r^{2}\right), \\
R^{\prime}(r) & =B\left(r^{\prime \prime}\right) \times\left(\rho_{3} r^{2}, \rho_{4} r^{2}\right),
\end{aligned}
$$

where $B(r)$ denotes the $n$-ball of radius $r$ and center 0 , and where

$$
\begin{gathered}
0<r^{\prime \prime}<r^{\prime}<r \\
\sigma_{1}<\tau_{1}<\rho_{1}<\rho_{2}<\tau_{2}<\tau_{3}<\rho_{3}<0<\rho_{4}<\tau_{4}<\sigma_{2} .
\end{gathered}
$$

Because we intend to employ both Theorems 2.1 and 3.1 to establish continuity of weak solutions of (1), we impose the following constraints on the geometry of the above cylinders in order that the hypotheses of Theorem 3.1 can be satisfied for a particular cylinder that will be specified later.

First, let $r^{\prime \prime}=\frac{1}{2} r$. Let $\omega$ be a number such that $0<\omega<2^{-1}$, and choose

$$
\rho_{1}=\frac{1}{4}\left(\frac{\omega}{2}-1\right), \quad \rho_{2}=-\frac{1}{4} \cdot \frac{\omega}{2} \text {. }
$$

Now choose $\tau_{1}<\rho_{1}, \rho_{2}<\tau_{2}<0$ and select $\tau_{3}, \tau_{4}$ so that $\tau_{2}<\tau_{3}<0, \tau_{4}>0$ and $\tau_{4}-\tau_{3}=\tau_{2}-\tau_{1}$. Next, choose $\tau_{3}<\rho_{3}<0$ and $0<\rho_{4}<\tau_{4}$. Finally, choose $\sigma_{1}<\tau_{1}$, $\tau_{4}<\sigma_{2}$ and note that $R((r / 2) N) \subset R^{\prime}(r)$, for some positive integer $N$. For $u$ defined on $\Omega_{T}$ and $z_{0} \in \Omega_{T}$, let

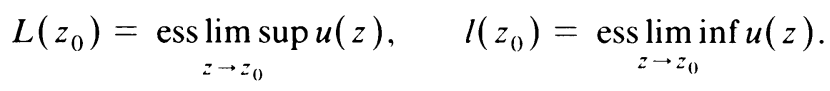

We can now state the main result in this section.

THEOREM 2.1. Let $u \in W_{2}^{1.1}\left(\Omega_{T}\right)$ be a bounded weak solution of (1) with structure (6), where $\beta$ is of either type I or type II. Then, for any $z_{0} \in \Omega_{T}$,

$$
\lim _{r \rightarrow 0} r^{-(n+2)} \int_{R^{*}(r)}\left|u-L\left(z_{0}\right)\right|=0, \quad \text { if } L\left(z_{0}\right)>0,
$$

or

$$
\lim _{r \rightarrow 0} r^{-(n+2)} \int_{R^{*}(r)}\left|u-l\left(z_{0}\right)\right|=0, \quad \text { if } l\left(z_{0}\right)<0
$$

The proof of this result follows the approach adopted in $[\mathbf{T}]$. Note that the result implies that $L\left(z_{0}\right)$ and $l\left(z_{0}\right)$ cannot have opposite signs. 
Proof. Take $z_{0}=(0,0)$ and assume that $L\left(z_{0}\right)>0$. For $k>0$, let $u_{k}=(u-k)^{+}$ and define

$$
\mu(r)=\operatorname{ess} \sup \{u(z): z \in R(r)\},
$$

where the notation of (6) is now being employed. Without loss of generality, we may assume that $\mu(r)>0$, for all small $r$, for otherwise we would consider $l\left(z_{0}\right)$. Then, the only interesting case would be $l\left(z_{0}\right)<0$ and therefore, $\lambda(r)>0$, for all $r>0$, where

$$
\lambda(r)=-\operatorname{essinf}\{u(z): z \in R(r)\} .
$$

In what follows, we would then choose $k<0$, replace $(u-k)^{+}$by $(u-k)^{-}$and replace $\mu(r)$ by $\lambda(r)$. The proof would remain essentially unchanged in all other aspects.

Now choose $0<k<L\left(z_{0}\right)$ and define $\mu_{k}(r)=\mu(r)-k$. For $\varepsilon>0$ and $\delta(r)=r$ $+d r+(d r)^{2}$ we define the function

$$
v(z)= \begin{cases}\mu_{k}(r)+\delta(r)-u_{k}(z), & \text { on }\{u \geqslant k\} ; \\ \mu_{k}(r)+\delta(r), & \text { on }\{u<k\} ;\end{cases}
$$

and consider as test functions in Definition 1.1,

$$
\phi=\eta^{2} \exp -\left(b_{0} u_{k}(z)\right)\left(v^{\alpha}-\left[\mu_{k}(r)+\delta(r)\right]^{\alpha}\right),
$$

where $\alpha<0$ and $\eta \geqslant 0$, is a smooth cut-off function that will be specified below. For the moment we assume only that spt $\eta \subset R(r) \subset \Omega_{T}$. Observe that $\phi=0$ on $\{u<k\}$. Because $u \in W_{2}^{1,1}\left(\Omega_{T}\right)$, it is well known that $u(x, \cdot)$ is an absolutely continuous function of $t$ for a.e. $x \in \Omega$. If $\beta$ is of type II, it follows from classical real analysis that $\beta\left[(u-k)^{+}+k\right](x, \cdot)$ is also absolutely continuous. Consequently, integration by parts allows identity (5) to be written as

$$
\int_{t_{0}}^{t_{1}} \int_{\Omega} w_{t} \phi+A\left(x, t, u, u_{x}\right) \cdot \phi_{x}+B\left(x, t, u, u_{x}\right) \phi=0,
$$

for all intervals $\left[t_{0}, t_{1}\right] \subset(0, T]$. In case $\beta$ is of type $\mathrm{I}$, it is easy to verify that (5) can be written as (9) with $w_{t}$ replaced by $u_{t}$. Thus, in either case, substitution of (8) into (9) yields

$$
\begin{gathered}
\int_{t_{0}}^{t_{1}} \int_{\Omega} w_{t} \phi+\int_{t_{0}}^{t_{1}} \int_{\Omega} \eta^{2} e^{-b_{0} u_{k}} \cdot\left[|\alpha| v^{\alpha-1}+b_{0}\left(v^{\alpha}-\left[\mu_{k}(r)+\delta(r)\right]^{\alpha}\right)\right] A \cdot u_{x} \\
=-\int_{t_{0}}^{t_{1}} \int_{\Omega} 2 \eta A \cdot \eta_{x} e^{-b_{0} u_{k}}\left(v^{\alpha}-\left[\mu_{k}(r)+\delta(r)\right]^{\alpha}\right)-\int_{t_{0}}^{t_{1}} \int_{\Omega} B \phi .
\end{gathered}
$$

Using $v^{\alpha}-\left[\mu_{k}(r)+\delta(r)\right]^{\alpha} \leqslant v^{\alpha}, v \geqslant \delta(r)$, the structure (6) and Young's inequality, it follows that there is a constant $C_{1}$ depending on the structure (6) such that

$$
\begin{gathered}
-|\alpha|^{-1} \int_{t_{0}}^{t_{1}} \int_{\Omega} \eta^{2} e^{-b_{0} u_{k}}\left\{v^{\alpha}-\left[\mu_{k}(r)+\delta(r)\right]^{\alpha}\right\} v \beta^{\prime}(u)+\int_{t_{0}}^{t_{1}} \int_{\Omega} \eta^{2} v^{\alpha-1}|\nabla v|^{2} \\
\leqslant C_{1}\left(1+|\alpha|^{-1}\right) \int_{t_{0}}^{t_{1}} \int_{\Omega}\left[\left(r^{-1} \eta\right)^{2}+|\nabla \eta|^{2}\right] v^{\alpha+1}
\end{gathered}
$$


This inequality holds in case $\beta$ is of type II. In the event $\beta$ is of type I, the inequality will be the same except $\beta^{\prime}(u)$ should be replaced by 1 . Note that the first term on the left is zero on the set $\{u<k\}$ and therefore $\beta^{\prime}(u)$ can be replaced by $\beta^{\prime}\left(u_{k}+k\right)$ in case $\beta$ is of type II. Let

$$
f(v)=\int_{0}^{v} e^{-h_{0}\left(\mu_{k}(r)+\delta(r)-s\right)} \beta^{\prime}\left(\mu_{k}+\delta(r)+k-s\right)\left[S^{\alpha}-\left(\mu_{k}+\delta\right)^{\alpha}\right] d s .
$$

Thus, the first term on the left side of the above inequality now becomes

$$
-|\alpha|^{-1} \int_{t_{0}}^{t_{1}} \int_{\Omega} \eta^{2} f(v)_{t} d x d t
$$

From elementary estimates it follows that there is a constant $C$ (depending on $k$ ) such that

$$
-C^{-1}\left(\frac{\alpha}{\alpha+1}\right) v^{\alpha+1} \leqslant f(v) \leqslant C(\alpha+1)^{-1} v^{\alpha+1} .
$$

As in [T] this immediately yields the following inequality:

$$
\begin{aligned}
& \sup _{t} \int[\eta v(x, t)]^{2} d x+\int_{t_{0}}^{t_{1}} \int_{\Omega} \eta^{2} v^{\alpha-1}|\nabla v|^{2} d x d t \\
& \quad \leqslant C\left(1+|\alpha|^{-1}\right)|1+\alpha|^{-1} \int_{t_{0}}^{t_{1}} \int_{\Omega}\left[\left(r^{-1} \eta\right)^{2}+|\nabla \eta|^{2}+\eta\left|\eta_{t}\right|\right] v^{\alpha+1} d x d t,
\end{aligned}
$$

where $C$ depends on $k$ and is bounded when $\alpha$ is bounded away from 0 and -1 . There is a similar inequality in case $\alpha=-1$. The dependency of the constant on $k$ is immaterial as $k$ is fixed throughout the proof. In case $\beta$ is of type I, the same inequality results except that $C$ does not depend on $k$. This inequality is the basis for an iteration procedure involving $\alpha$ which leads to the desired result. Details will not be given, for the argument is similar to that given in [T] or [AS]. For complete details involving the general structure (2), see [GZ2].

We will complete the proof of Theorem 2.1 by indicating the main points of the iteration scheme. For $p$ any extended real number and $R$ a cylinder, let

$$
A(p, R)=\left(|R|^{-1} \int_{R} v^{p}\right)^{1 / p}
$$

where $|R|$ denotes the $(n+1)$-Lebesgue measure of $R$. Thus, $A\left(-\infty, R^{\prime}(r)\right)$ denotes the infimum of $u$ over the cylinder $R^{\prime}(r)$. The Sobolev inequality along with (10) and the iteration scheme yields

$$
A\left(-p_{0}, R^{+}(r)\right) \leqslant C A\left(-\infty, R^{\prime}(r)\right), \quad A\left(p_{1}, R^{*}(r)\right) \leqslant C A\left(p_{0}, R^{-}(r)\right),
$$

for any $p_{0}>0, p_{1}>1$. In case $\alpha=-1$, the John-Nirenberg lemma implies that there exists $p^{*}>0$ such that

$$
A\left(p^{*}, R^{-}(r)\right) \leqslant C A\left(-p^{*}, R^{+}(r)\right)
$$

Hence, from (10)

$$
\left(\left|R^{*}(r)\right|^{-1} \int_{R^{*}(r)} v^{p_{1}}\right)^{1 / p_{1}} \leqslant C \min _{R^{\prime}(r)} v
$$


or

$$
\begin{aligned}
& \left|R^{*}(r)\right|^{-1} \int_{R^{*}(r)}\left[\mu_{k}(r)+\delta(r)-u_{k}\right]^{p_{1}} \\
& \quad \leqslant C\left[\mu_{k}(r)+\delta(r)-\mu_{k}\left(r / 2^{N}\right)\right]^{p_{1}} .
\end{aligned}
$$

Observe that the right side of (12) tends to 0 as $r \downarrow 0$.

$$
\begin{aligned}
\int_{R^{*}(r)}\left[\mu_{k}(r)\right. & \left.+\delta(r)-u_{k}\right]^{p_{1}} \\
= & \int_{A^{*}(r, k)}[\mu(r)+\delta(r)-u]^{p_{1}}+\int_{B^{*}(r, k)}[\mu(r)-k+\delta(r)]^{p_{1}},
\end{aligned}
$$

where $A^{*}(r, k)=R^{*}(r) \cap\{z: u(z) \geqslant k\}$ and $B^{*}(k, r)=R^{*}(r)-A^{*}(r, k)$. Since $\mu(r) \rightarrow L\left(z_{0}\right)$ and $0<k<L\left(z_{0}\right)$, it follows from (12) and (13) that

$$
\left|B^{*}(r, k)\right| /\left|R^{*}(r)\right| \rightarrow 0, \quad \text { as } r \downarrow 0,
$$

and therefore that

$$
\left|R^{*}(r)\right|^{-1} \int_{B^{*}(r, k)}[\mu(r)+\delta(r)-u]^{p_{1}} \rightarrow 0, \quad \text { as } r \downarrow 0 .
$$

Since $\delta(r) \rightarrow 0, \mu(r) \rightarrow L\left(z_{0}\right)$ and $\left|R^{*}(r)\right|=c r^{n+2},(12),(13)$ and (14) imply

$$
\lim _{r \rightarrow 0} r^{-(n+2)} \int_{R^{*}(r)}\left|u-L\left(z_{0}\right)\right|^{p_{1}}=0 .
$$

3. A maximum principle. In this section we prove a lemma which is fundamental to the theory of regularity as developed by DeGiorgi. Nondegenerate elliptic and parabolic versions of this lemma appear in [LU, p. 83] and [LSU, p. 114]. The lemma was also employed in [GZ1] for the purpose of establishing boundary regularity of weak solutions of nondegenerate elliptic equations. In the case of the two-phase Stefan problem, a version of this lemma is critical to the proof of continuity given by Caffarelli and Evans, [CE, Lemma 3.1]. DiBenedetto, [D1, D2], extended the lemma as it appears in [LSU] to weak solutions of (1) with general structure (2). For the convenience of the reader we will also include a brief proof. For the sake of brevity, we will assume the restricted structure (6) rather than (2).

Throughout this section we let $\left(x_{1}, t_{1}\right) \in \Omega_{T}, t_{1}>0$ and for $r>0$ we let $Q(r)$ denote the cylinder

$$
Q(r)=B\left(x_{1}, r\right) \times\left[t_{1}-r^{2}, t_{1}\right],
$$

where $B\left(x_{1}, r\right)$ denotes the $n$-ball of radius $r$ and center $x_{1}$. We will consider only those cylinders for which $Q(r) \subset \Omega_{T}$. In case $\beta$ is of type II, we will assume that $a$ bounded solution $u \in V_{2}\left(Q_{T}\right)$ of (1) is the weak $V_{2}\left(Q_{T}\right)$ limit of a uniformly bounded sequence $\left\{u_{n}\right\}$, where each $u_{n}$ is a weak solution of (1) in which $\beta$ is replaced by a $C^{\infty}\left(R^{1}\right)$ function $\beta_{n}$. The $\beta_{n}$ are chosen so that $\beta_{n}(0)=0$,

$$
0<\alpha_{0} \leqslant \beta_{n}^{\prime}(r) \leqslant \beta^{\prime}(r), \quad r \neq 0, \quad n=1,2, \ldots,
$$


and that $\beta_{n} \rightarrow \beta$ uniformly on compact subsets of $R^{1}$. This assumption is in accord with results concerning existence of weak solutions of (1), $\mathrm{cf}$. [CD].

We also assume for simplicity that solutions $u$ of (1) are elements of $W_{2}^{1,1}\left(\Omega_{T}\right)$. In case $\beta$ is of type II this is no loss of generality in view of standard techniques involving integral averages. Let $m(r)=\operatorname{ess} \sup \{u(z): z \in Q(r)\}$ and for $k \in R^{1}$, let

$$
A(k, r)=Q(r) \cap\{z: u(z) \geqslant k\} .
$$

Lemma 3.1. Let $u \in W_{2}^{1,1}\left(\Omega_{T}\right)$ be a bounded, weak solution of (1). There exist positive numbers $\theta, \kappa$ and $\delta$ depending only on the general structure of (1) such that if $k \in R^{1}$ and

$$
r_{0}^{(n / 2) \kappa}+k<m\left(r_{0}\right), \quad m\left(r_{0}\right)<k+\delta, \quad \text { and }\left|A\left(k, r_{0}\right)\right|<\theta\left|Q\left(r_{0}\right)\right|,
$$

then

$$
\left|A\left[2^{-1}\left(m\left(r_{0}\right)+k\right), r_{0} / 2\right]\right|=0
$$

Proof. First, assume that $\beta$ is of type I and choose a function $w \subset \beta(u)$. Define

$$
\sigma(z)= \begin{cases}a, & \text { if } u(z)>0 \\ w(z)+a, & \text { if } u(z)=0 \\ 0, & \text { if } u(z)<0\end{cases}
$$

and note that $w+a=u+\sigma$. Thus, substituting $w$ into (5) yields

$$
\begin{gathered}
-\left.\int \sigma(x, t) \phi(x, t) d x\right|_{t_{0}} ^{t_{1}}+\int_{t_{0}}^{t_{1}} \int_{\Omega} \sigma(x, t) \phi_{t}(x, t) d x d t \\
=\int_{t_{0}}^{t_{1}} \int_{\Omega} A \cdot \nabla \phi+B \phi+u_{t} \phi d x d t
\end{gathered}
$$

Let $\phi=\eta^{2}(u-k)^{+}$where $k<0$ and $\eta$ is a smooth cut-off function that will be specified below. Choose $\eta$ so that $\eta_{t} \geqslant 0$ and $\eta$ vanishes at $t_{0}$. Then

$$
\begin{aligned}
-\left.\int \sigma(x, t) \phi(x, t) d x\right|_{t_{0}} ^{t_{1}} & =-\left.\int \sigma u^{+} \eta^{2} d x\right|_{t_{0}} ^{t_{1}}+\left.k \int \sigma \eta^{2} d x\right|_{t_{0}} ^{t_{1}} \\
& \leqslant-\left.\int \sigma u^{+} \eta^{2} d x\right|_{t_{0}} ^{t_{1}} .
\end{aligned}
$$

Also,

$$
\int_{t_{0}}^{t_{1}} \int_{\Omega} \sigma \phi_{t} d x d t=\int_{t_{0}}^{t_{1}} \int_{\Omega} \sigma u_{t}^{+} \eta^{2} d x d t+2 \int_{t_{0}}^{t_{1}} \int_{\Omega} \eta \eta_{t}(u-k)^{+} \sigma d x d t,
$$

and therefore

$$
\int_{t_{0}}^{t_{1}} \int_{\Omega} A \cdot \nabla \phi+B \phi+u_{t} \phi d x d t \leqslant \int_{t_{0}}^{t_{1}} \int_{\Omega} 2 \sigma \eta \eta_{t}(u-k)^{+} d x d t
$$


Therefore, from (6) and elementary estimates we have

$$
\begin{aligned}
& \sup _{t_{0} \leqslant t \leqslant t_{1}} \int_{\Omega}\left[\eta(u-k)^{+}\right]^{2} d x+\int_{t_{0}}^{t_{1}} \int_{\Omega} \mid \nabla\left[\eta(u-k)^{+}\right]^{2} d x d t \\
& \leqslant C\left(\int_{t_{0}}^{t_{1}} \int_{\Omega}(u-k)^{+2}\left(|\nabla \eta|^{2}+\eta \eta_{t}\right)\right. \\
& \left.\quad+\int_{t_{0}}^{t_{1}} \int_{\Omega}(u-k)^{+} \eta \eta_{t} d x d t+\iint \eta^{2} \chi(u \geqslant k)\right),
\end{aligned}
$$

provided $\eta$ vanishes at $t_{0}$. Here $\chi(E)$ denotes the characteristic function of a set $E$. If $k \geqslant 0$ this estimate is even easier to establish. In case $\beta$ is of type II, the estimate is easily seen to be valid for the approximating $\beta_{n}$ and passage to the limit yields the estimate for $\beta$.

Now choose $k \in R^{1}$ and let $H=m\left(r_{0}\right)-k$. For each positive integer $m$ let

$$
\begin{gathered}
k_{m}=\left(k+\frac{1}{2} H\right)-H / 2^{m}, \quad r_{m}=\left(r_{0} / 2\right)\left(1+1 / 2^{m}\right), \\
Q_{m}=B\left(x_{1}, r_{m}\right) \times\left[t_{1}-r_{m}^{2}, t_{1}\right],
\end{gathered}
$$

and choose smooth cut-off functions $\eta_{m}$ such that $\eta_{m}=1$ on $Q_{m+1}, \eta_{m}=0$ near the parabolic boundary of $Q_{m},\left|\nabla \eta_{m}\right| \leqslant C 2^{m} / r_{0}$ and $0 \leqslant \partial \eta_{m} / \partial t \leqslant C 4^{m} / r_{0}^{2}$. Substituting $k=k_{m+1}$ and $\eta=\eta_{m}$ into the previous inequality, we have from the parabolic Sobolev inequality, vide [LSU, p. 75],

$$
\begin{aligned}
& \left(\int_{Q_{m+1}} \int\left(u-k_{m+1}\right)^{+2 \sigma}\right)^{1 / \sigma} \\
& \quad \leqslant \frac{C 8^{m}}{r_{0}^{2}}\left[\int_{Q_{m}} \int\left(u-k_{m}\right)^{+^{2}}+\int_{Q_{m}} \int\left(u-k_{m+1}\right)^{+}\right]+\left|Q_{m} \cap\left\{u>k_{m+1}\right\}\right|,
\end{aligned}
$$

where $\sigma=(n+2) / n$. Observe that

$$
\begin{gathered}
\int_{Q_{m}} \int\left(u-k_{m+1}\right)^{+} \leqslant \frac{C(m+1)^{2}}{H} \int_{Q_{m}} \int\left(u-k_{m}\right)^{+^{2}}, \\
\left|Q_{m} \cap\left\{u>k_{m+1}\right\}\right| \leqslant \frac{C(m+1)^{4}}{H^{2}} \int_{Q_{m}} \int\left(u-k_{m}\right)^{+^{2}},
\end{gathered}
$$

and

$$
\int_{Q_{m+1}} \int\left(u-k_{m+1}\right)^{+^{2}} \leqslant\left(\int_{Q_{m+1}} \int\left(u-k_{m+1}\right)^{+^{20}}\right)^{1 / \sigma}\left|Q_{m} \cap\left\{u>k_{m+1}\right\}\right|^{2 /(n+2)}
$$

Thus if we let

$$
I_{m}=\int_{Q_{m}} \int\left(u-k_{m}\right)^{+^{2}}
$$

it follows that

$$
I_{m+1} \leqslant \frac{C C_{1}^{m} 8^{m}}{r_{0}^{2}}\left(H^{2}+H+r_{0}^{2}\right)\left(\frac{I_{m}}{H^{2}}\right)^{(n+4) /(n+2)}
$$


Therefore,

$$
\frac{I_{m+1}}{r_{0}^{n+2} H^{2}} \leqslant C C_{1}^{m} 8^{m}\left[1+\frac{1}{H}+\left(\frac{r_{0}}{H}\right)^{2}\right]\left(\frac{I_{m}}{r_{0}^{n+2} H^{2}}\right)^{(n+4) /(n+2)} .
$$

With general structure (2), it can be shown that $\kappa<2 / m$. Thus, for small $r_{0}$, we have from hypothesis that $r_{0}^{2}<H^{2}$ and because $u$ is assumed to be bounded, there is a constant depending on $\sup |u|$ such that $1<C / H$, and therefore

$$
J_{m+1} \leqslant \frac{C_{1}^{m} 8^{m}}{H}\left(J_{m}\right)^{(n+4) /(n+2)},
$$

where $J_{m}=I_{m} / r_{0}^{n+2} H^{2}$. According to [LU, p. 66], $J_{m} \rightarrow 0$ as $m \rightarrow \infty$ if $J_{1}$ is sufficiently small. However,

$$
J_{1} \leqslant C\left|A\left(k, r_{0}\right)\right| / r_{0}^{n+2}
$$

and thus, the conclusion now follows.

Continuity of weak solutions of (1) readily follow from Theorem 2.1 and Lemma 3.1. To see this, choose $z_{0} \in \Omega_{T}$ and as in Theorem 2.1 , take $z_{0}=(0,0)$ and assume $l\left(z_{0}\right)<0$. Then it follows from Theorem 2.1 that

$$
r^{-(n+2)} \int_{R^{*}(r)}\left|u-l\left(z_{0}\right)\right| \rightarrow 0, \quad \text { as } r \rightarrow 0 .
$$

Referring to (7) we have that

$$
R^{*}(r)=B\left(\frac{r}{2}\right) \times\left[\left(\frac{\omega-2}{2}\right)\left(\frac{r}{2}\right)^{2},-\frac{\omega}{2}\left(\frac{r}{2}\right)^{2}\right],
$$

where $0<\omega<\frac{1}{2}$. Define

$$
\begin{aligned}
Q\left(\frac{r}{2}\right) & =B\left(\frac{r}{2}\right) \times\left[\left(\frac{\omega-2}{2}\right)\left(\frac{r}{2}\right)^{2}, \frac{\omega}{2}\left(\frac{r}{2}\right)^{2}\right] \\
& =B\left(\frac{r}{2}\right) \times\left[t_{1}-\left(\frac{r}{2}\right)^{2}, t_{1}\right]
\end{aligned}
$$

where $t_{1}=\omega r^{2} / 8$.

Suppose that $u$ is not continuous at $z_{0}$, i.e., that $l\left(z_{0}\right)<L\left(z_{0}\right)$. Select a number $k$ and $\delta^{*} \leqslant \delta$ such that

$$
L\left(z_{0}\right)-\frac{\delta^{*}}{2}>k>L\left(z_{0}\right)-\frac{3}{4} \delta^{*}>l\left(z_{0}\right) .
$$

Choose $r$ small enough so that

$$
m\left(\frac{r}{2}\right)<L\left(z_{0}\right)+\frac{1}{4} \delta^{*}, \quad\left(\frac{r}{2}\right)^{n / 2^{\kappa}}+k<m\left(\frac{r}{2}\right),
$$

and therefore that

$$
m(r / 2)<k+\delta, \quad m(r / 2)+k<2 L\left(z_{0}\right) .
$$

It follows from (15) that

$$
\left|R^{*}(r) \cap\{z: u(z) \geqslant k\}\right|<(\theta / 2)\left|R^{*}(r)\right|
$$


for all small $r$, and therefore

$$
|A(k, r / 2)|=|Q(r / 2) \cap\{z: u(z) \geqslant k\}|<(\theta / 2+\omega)|Q(r / 2)| \text {. }
$$

Thus, if $2 \omega<\min (\theta, 1)$ and if $r$ is small enough to satisfy (16), (17) and (18), we see that the hypotheses of Lemma 3.1 are satisfied with $r_{0}=r / 2$. Note that

$$
Q\left(r_{0} / 2\right)=B(r / 4) \times\left[t_{1}-(r / 4)^{2}, t_{1}\right]
$$

and $t_{1}-(r / 4)^{2}=\left(r^{2} / 16\right)(2 \omega-1)<0$. Therefore, $z_{0} \in Q\left(r_{0} / 2\right)$. However, (17) and Lemma 2.1 imply that $m\left(r_{0} / 2\right)<L\left(z_{0}\right)$, a contradiction.

Similar reasoning can be applied if we assume $l\left(z_{0}\right)<L\left(z_{0}\right)$ with $L\left(z_{0}\right)>0$. However, in this case a statement dual to Lemma 2.1, which involves the measure of $\{z: u(z) \leqslant k\}$, would have to be employed. Thus, we have shown that $l\left(z_{0}\right)=L\left(z_{0}\right)$ for every $z_{0} \in \Omega_{T}$. Throughout the proof, we have tacitly assumed that $0<t_{0}<T$ where $z_{0}=\left(x_{0}, t_{0}\right)$. The reader can verify that the proof can be easily modified to accommodate the case $t_{0}=T$. Therefore we have the following.

THEOREM 3.2. Suppose $u \in W_{2}^{1,1}\left(\Omega_{T}\right)$ is a bounded solution of (1) where $\beta$ is of type I or of type II subject to assumptions of this section. Then, after redefinition on a set of measure zero, $u$ is continuous on $\Omega_{T}$.

The author is indebted to the referee who observed that it is possible to obtain a modulus of continuity for weak solutions by performing a more detailed analysis of inequality (12).

4. Continuity at the boundary. In this section, we consider the question of continuity of weak solutions at a boundary point $z_{0} \in \partial \Omega \times(0, T)$. We consider a function $f$ defined on all of $R^{n+1}$ such that

$$
f \in W_{2}^{1,1}\left(R^{n+1}\right) \text { and } f \mid R^{n+1}-\Omega \text { is continuous. }
$$

Throughout this section we will consider a bounded weak solution $u \in W_{2}^{1,1}\left(\Omega_{T}\right)$ of (1) such that

$$
u=f \text { weakly on } \partial \Omega_{T} ;
$$

that is, $u-f$ is assumed to be in the $W_{2}^{1,1}\left(\Omega_{T}\right)$-closure of smooth functions $\psi$ where spt $\psi \subset \Omega_{T}$. If $\beta$ is of type II we assume, as in $\S 3$, that a bounded solution $u$ of (1), whose trace is $f$ on the lateral boundary of $\Omega_{T}$, is the $V_{2}$ limit of a uniformly bounded sequence $\left\{u_{n}\right\}$, where each $u_{n}$ is a weak solution of (1) in which $\beta$ is replaced by $\beta_{n}$ and where the trace of $u_{n}$ on the lateral boundary is $f$. Define

$$
L\left(\Omega_{T}, z_{0}\right)=\limsup _{z \rightarrow z_{0}} u(z), \quad l\left(\Omega_{T}, z_{0}\right)=\liminf _{z \rightarrow z_{0}} u(z) .
$$

Because we are assuming that $u=f$ weakly on $\partial \Omega_{T}$, it will be convenient to extend $u$ to all of $R^{n+1}$ by setting

$$
u= \begin{cases}u, & \text { on } \Omega_{T}, \\ f, & \text { on } R^{n+1}-\Omega_{T} .\end{cases}
$$


Thus, $u$ can now be considered as an element of $W_{2}^{1,1}\left(R^{n+1}\right)$ and therefore

$$
\lim _{r \rightarrow 0} r^{-(n+2)} \int_{W\left(z_{0}, r\right)}\left|u(z)-u\left(z_{0}\right)\right| d z=0
$$

for all $z_{0}=\left(x_{0}, t_{0}\right) \in R^{n+1}$, except perhaps for a set of Newtonian capacity zero, see $[\mathbf{D Z}]$. Here $W\left(z_{0}, r\right)$ denotes the parabolic cylinder

$$
B\left(x_{0}, r\right) \times\left[t_{1}-r^{2}, t_{1}\right],
$$

where $t_{1}=t_{0}+r^{2} / 8$.

In order to facilitate the proof of boundary regularity we need

DEFINITION 4.1. For an arbitrary set $E \subset R^{n+1}$, we define a capacity by

$$
\Gamma(E)=\inf \left\{\underset{t}{\operatorname{ess} \sup } \int u(x, t)^{2} d x+\iint|\nabla u|^{2} d x d t\right\},
$$

where the infimum is taken over all functions $u \in W_{2}^{1,1}\left(R^{n+1}\right)$ for which

$$
E \subset \operatorname{int}\{z: u(z) \geqslant 1\} \text {. }
$$

Recall that the Newtonian capacity of a set $A \subset R^{n}$ can be defined as

$$
\gamma(A)=\inf \left\{\int|\nabla u|^{2}\right\}
$$

where the infimum is taken over all $u \in W_{2}^{1}\left(R^{n}\right)$ such that $A \subset \operatorname{int}\{x: u(x) \geqslant 1\}$. It is well known that $\gamma[B(r)]=C r^{n-2}$. As an immediate consequence of the definitions, we have the estimate

$$
\Gamma(E) \geqslant \int_{-\infty}^{\infty} \gamma[E(t)] d t+\underset{t}{\operatorname{ess} \sup }|E(t)| .
$$

Now suppose that $E=A \times(0, T]$, where $A \subset B\left(x_{0}, a\right)$ for some $x_{0} \in R^{n}$ and $a \in R^{1}$. It follows from elementary estimates that there are constants $C_{1}, C_{2}$ such that

$$
\Gamma(E) \leqslant C_{1} T \gamma(A)+C_{2} a^{2} \gamma(A) .
$$

In particular, if $E$ is taken as a parabolic cylinder $R(r)$ (see (7)), then (23) and (24) imply that $\Gamma[R(r)] \sim r^{n}$.

It will be shown below that a weak solution of (1) is continuous at $z_{0}$ if

$$
\int_{0}^{1} \frac{\Gamma\left[R^{*}(r)-\Omega_{T}\right]}{\Gamma\left[R^{*}(r)\right]} \frac{d r}{r}=\infty .
$$

Because $\Omega_{T}=\Omega \times(0, T],(23)$ and (24) imply

$$
\Gamma\left[R^{*}(r)-\Omega_{T}\right] \leqslant C_{1} r^{2} \gamma\left[B\left(x_{0}, r\right)-\Omega\right]+C_{2} r^{2} \gamma\left[B\left(x_{0}, r\right)-\Omega\right]
$$

and

$$
\Gamma\left[R^{*}(r)-\Omega_{T}\right] \geqslant(r / 2)^{2} \gamma\left[B\left(x_{0}, r / 2\right)-\Omega\right] .
$$

Since $\Gamma\left[R^{*}(r)\right] \sim r^{n}$ and $\gamma\left[B\left(x_{0}, r\right)\right] \sim r^{n-2},(25)$ holds if and only if

$$
\int_{0}^{1} \frac{\gamma\left[B\left(x_{0}, r\right)-\Omega\right]}{\gamma\left[B\left(x_{0}, r\right)\right]} \frac{d r}{r}=\infty
$$

which is the Wiener criterion for regularity at $x_{0} \in \partial \Omega$. 
We now consider parabolic cylinders

$$
Q(r)=B\left(x_{1}, r\right) \times\left[t_{1}-r^{2}, t_{1}\right]
$$

where $\left(x_{1}, t_{1}\right) \in \partial \Omega \times(0, T)$ and let

$$
m\left(\Omega_{T}, r\right)=\sup \left\{u(z): z \in \Omega_{T} \cap Q(r)\right\} .
$$

The proof of the following is almost identical to that of Lemma 2.1.

LEMMA 4.1. Let $u \in W_{2}^{1,1}\left(\Omega_{T}\right)$ be a bounded weak solution of (1) such that $u=f$ weakly on $\partial \Omega_{T}$. There exist positive numbers $\theta, \kappa$ and $\delta$ depending only on the general structure of (1) such that if

$$
\begin{gathered}
k \geqslant \sup \left\{f(z): z \in Q\left(r_{0}\right)-\Omega_{T}\right\}, \quad r_{0}^{(n / 2) \kappa}+k<m\left(\Omega_{T}, r_{0}\right), \\
m\left(\Omega_{T}, r_{0}\right)<k+\delta \text { and }\left|A(k, r) \cap \Omega_{T}\right|<\theta\left|Q\left(r_{0}\right)\right|,
\end{gathered}
$$

then

$$
\left|A\left[2^{-1}\left(m\left(\Omega_{T}, r_{0}\right)+k\right), r_{0} / 2\right] \cap \Omega_{T}\right|=0
$$

Lemma 4.2. Suppose $z_{0}=\left(x_{0}, t_{0}\right) \in \partial \Omega \times(0, T)$ is such that

$$
\liminf _{r \rightarrow 0} \frac{\left|W\left(z_{0}, r\right) \cap \Omega_{T}\right|}{\left|W\left(z_{0}, r\right)\right|}=0 .
$$

Then

$$
\lim _{\substack{z \rightarrow z_{0} \\ z \in \Omega_{T}}} u(z)=f\left(z_{0}\right)
$$

Proof. It suffices to prove $L\left(\Omega_{T}, z_{0}\right) \leqslant f\left(z_{0}\right)$ for the proof that $f\left(z_{0}\right) \leqslant l\left(\Omega_{T}, z_{0}\right)$ is similar. Consider parabolic cylinders of the form

$$
Q(r)=W\left(z_{0}, r\right), \quad\left|Q(r) \cap \Omega_{T}\right|<\theta|Q(r)| .
$$

Suppose $L\left(\Omega_{T}, z_{0}\right)>f\left(z_{0}\right)$ and choose $k$ and $\delta^{*} \leqslant \delta$, such that $L\left(\Omega_{T}, z_{0}\right)-\delta^{*} / 2$ $>k>L\left(\Omega_{T}, z_{0}\right)-(3 / 4) \delta^{*}>f\left(z_{0}\right)$. Now choose an $r_{0}$ so that

$$
\begin{gathered}
k \geqslant \sup \left\{f(z): z \in Q\left(r_{0}\right)-\Omega_{T}\right\}, \\
m\left(\Omega_{T}, r_{0}\right)<L\left(\Omega_{T}, z_{0}\right)+\frac{1}{4} \delta^{*}, \quad r_{0}^{(n / 2) \kappa}+k<m\left(\Omega_{T}, r_{0}\right),
\end{gathered}
$$

and therefore that

$$
m\left(\Omega_{T}, r_{0}\right)<k+\delta^{*}, \quad m\left(\Omega_{T}, r_{0}\right)+k<2 L\left(\Omega_{T}, z_{0}\right) .
$$

However, $z_{0} \in Q\left(r_{0} / 2\right)$ and therefore Lemma 4.1 implies

$$
L\left(\Omega_{T}, z_{0}\right)<\frac{1}{2}\left[m\left(\Omega_{T}, r_{0}\right)+k\right],
$$

a contradiction.

Consider a point $z_{0} \in \partial \Omega \times(0, T)$ at which (21) holds. In view of Lemma 4.2 it follows that

$$
L\left(\Omega_{T}, z_{0}\right) \geqslant f\left(z_{0}\right) \geqslant l\left(\Omega_{T}, z_{0}\right),
$$


for if not, then $L\left(\Omega_{T}, z_{0}\right)<f\left(z_{0}\right)-\varepsilon$ for some $\varepsilon>0$. Consequently, $u(z)<f\left(z_{0}\right)-$ $\varepsilon / 2$ for $z \in \Omega_{T} \cap W\left(z_{0}, r\right)$ and all small $r$. But (21) implies that

$$
\lim _{r \rightarrow 0} \frac{\left|W\left(z_{0}, r\right) \cap \Omega_{T}\right|}{\left|W\left(z_{0}, r\right)\right|}=0,
$$

thus contradicting Lemma 4.2. A similar argument shows that $f\left(z_{0}\right) \geqslant l\left(\Omega_{T}, z_{0}\right)$. Thus, (27) holds for all $z_{0} \in \partial \Omega \times(0, T)$ except for a set $S$ of Newtonian capacity zero. Let

$$
\begin{gathered}
A=S \cap\left\{z: W(z, r)-\Omega_{T} \subset S, \text { for some } r>0\right\}, \quad \text { and } \\
B=S-A .
\end{gathered}
$$

Observe $W(z, r)-\Omega_{T}$ is a set of the form $W(z, r)-\Omega_{T}=(B(x, r)-\Omega) \times I$ where $I$ is an interval. As $S$ has zero Newtonian capacity in $R^{n+1}$, it follows that $\gamma[B(x, r)-\Omega]=0$. Thus, from (25) and (26) we have the

$$
\int_{0}^{1} \frac{\Gamma\left[R^{*}(r)-\Omega_{T}\right]}{\Gamma\left[R^{*}(r)\right]} \frac{d r}{r}=0<\infty,
$$

for all $z \in A$. Note that points of $B$ are limits of points at which (27) holds because $S$ is of Newtonian capacity zero, and therefore has Hausdorff dimension at most $n-1$ in $R^{n+1}$. As $f$ is continuous on $R^{n+1}-\Omega_{T}$, it follows that (27) holds at all points of $B$. Thus, $B$ is empty and

$$
L\left(\Omega_{T}, z_{0}\right) \geqslant f\left(z_{0}\right) \geqslant l\left(\Omega_{T}, z_{0}\right)
$$

at all $z_{0} \in \partial \Omega \times(0, T)$, except possibly at those where (29) holds.

To complete the proof of regularity at $z_{0} \in(\partial \Omega) \times(0, T)$, we first prove a result similar to Theorem 2.1. To this end let $z_{0}$ be a point at which (30) holds, assume $u=f$ weakly and let $f\left(z_{0}\right) \geqslant 0$. For $k>f\left(z_{0}\right)$ let

$$
\begin{aligned}
& u_{k}(z)= \begin{cases}(u(z)-k)^{+}, & z \in \Omega_{T}, \\
0, & z \notin \Omega_{T} ;\end{cases} \\
& \mu_{k}(r)=\sup \left\{u_{k}(z): z \in R(r)\right\} ;
\end{aligned}
$$

and define

$$
v(z)= \begin{cases}\mu_{k}(r)+\delta(r)-u_{k}(z), & z \in R(r) \cap \Omega_{T}, \\ \mu_{k}(r)+\delta(r), & z \in R(r)-\Omega_{T} .\end{cases}
$$

Proceeding exactly as in the proof of Theorem 2.1, we have

$$
\left(r^{-(n+2)} \int_{R^{*}(r)} v^{p_{1}}\right)^{1 / p_{1}} \leqslant C \min _{R^{\prime}(r)} v
$$

for some $p_{1}>1$, and therefore

$$
r^{-(n+2)} \int_{R^{*}(r) \cap \Omega_{T}}\left|u-L\left(\Omega_{T}, z_{0}\right)\right|^{p_{1}} \rightarrow 0
$$


Observe that a similar argument yields

$$
r^{-(n+2)} \int_{R^{*}(r) \cap \Omega_{T}}\left|u-l\left(\Omega_{T}, z_{0}\right)\right|^{p_{1}} \rightarrow 0, \quad \text { as } r \downarrow 0
$$

if $l\left(\Omega_{T}, z_{0}\right)<f\left(z_{0}\right) \leqslant 0$.

If $f\left(z_{0}\right) \geqslant 0$, condition (30) allows us to treat (1) as though it were a nondegenerate equation whenever the test function $\phi$ in (8) involves $(u-k)^{+}$for $k>f\left(z_{0}\right)$ or $(u-k)^{-}$for $k<f\left(z_{0}\right) \leqslant 0$. For this reason the results of [Z] carry over to the present situation with essentially no change and thus yield the following.

TheOREM 4.3. Suppose (25) holds at $z_{0} \in \partial \Omega \times(0, T)$. Then $L\left(\Omega_{T}, z_{0}\right)=f\left(z_{0}\right)$ if $f\left(z_{0}\right) \geqslant 0$ and $l\left(\Omega_{T}, z_{0}\right)=f\left(z_{0}\right)$ if $f\left(z_{0}\right) \leqslant 0$.

Observe that the conclusions of Theorem 4.3 follow immediately from (31) if a stronger condition than (25) is assumed to hold at $z_{0}=\left(x_{0}, t_{0}\right)$, namely, if $R^{n}-\Omega$ is assumed to have positive upper metric density at $x_{0}$, i.e., if

$$
\limsup _{r \rightarrow 0} \frac{\left|B\left(x_{0}, r\right) \cap\left(R^{n}-\Omega\right)\right|}{\left|B\left(x_{0}, r\right)\right|}>0 .
$$

Now by employing Lemma 4.1, Theorem 4.3 and the argument used to establish Theorem 3.2, we have

THEOREM 4.4. Let $u \in W_{2}^{1,1}\left(\Omega_{T}\right)$ be a bounded weak solution of (1) such that $u=f$ weakly on $\partial \Omega_{T}$. We assume $\beta$ to be of type I or of type II. If (25) holds at $z_{0} \in \partial \Omega \times(0, T)$, then

$$
\lim _{\substack{z \rightarrow z_{0} \\ z \in \Omega_{T}}} u(z)=f\left(z_{0}\right)
$$

\section{REFERENCES}

[AS] D. G. Aronson and J. Serrin, Local behavior of solutions of quasilinear parabolic equations, Arch. Rational Mech. Anal. 25 (1967), 81-123.

[CD] J. R. Cannon and E. DiBenedetto, On the existence of weak solutions to an $n$-dimensional Stefan problem with non-linear boundary conditions, SIAM J. Math. Anal. 11 (1980).

[CE] L. A. Caffarelli and L. C. Evans, Continuity of the temperature in the two-phase Stefan problem (to appear).

[CF] L. A. Cafarelli and A. Friedman, Continuity of the density of a gas flow in a porous medium (to appear).

[D1] E. DiBenedetto, Continuity of weak solutions to certain singular parabolic equations (to appear).

[D2] ___ Continuity of weak solutions to a general porous media equation (to appear).

[DZ] D. J. Deignan and W. P. Ziemer, Strong differentiability properties of Bessel potentials, Trans. Amer. Math. Soc. 225 (1977), 113-122.

[GZ1] R. Gariepy and W. P. Ziemer, Behavior at the boundary of solutions of quasilinear elliptic equations, Arch. Rational Mech. Anal. 56 (1974), 372-384.

[GZ2] , A regularity condition at the boundary for solutions of quasilinear elliptic equations, Arch.

Rational Mech. Anal. 67 (1977), 25-39.

[LSU] O. A. Ladyzhenskaya, V. A. Solonnikov and N. N. Uraltseva, Linear and quasilinear equations of parabolic type, Transl. Math. Mono., vol. 23, Amer. Math. Soc., Providence, R. I., 1968. 
[LU] O. A. Ladyzhenskaya and N. N. Uraltseva, Linear and quasilinear elliptic equations, Academic Press, New York, 1968.

[S] P. Sacks, Existence and regularity of solutions of the inhomogeneous porous medium equations, Math. Research Center, Technical report, December, 1980.

[T] N. Trudinger, Pointwise estimates and quasilinear parabolic equations, Comm. Pure Appl. Math. 21 (1968), 205-266.

[Z] W. P. Ziemer, Behavior at the boundary of solutions of quasilinear parabolic equations, J. Differential Equations 35 (1980), 291-305.

Department of Mathematics, Indiana University, Bloomington, Indiana 47405 